\title{
From Sardinia to Japan: an update on the role of MAP in multiple sclerosis
}

\author{
Cossu Davide ${ }^{1,2}$ (D), Kazumasa Yokoyama1 ${ }^{1}$ Hattori Nobutaka ${ }^{1}$ \& Leonardo A Sechi*,3 (D) \\ ${ }^{1}$ Department of Neurology, Juntendo University, Tokyo, Japan \\ ${ }^{2}$ Advanced Research Institute for Health Science, Juntendo University, Tokyo, Japan \\ ${ }^{3}$ Microbiology Section, Department of Biomedical Sciences, University of Sassari, Italy \\ *Author for correspondence: Tel.: +39 079228 462; Fax: +39 079212 345; sechila@uniss.it
}

\begin{abstract}
' molecular mimicry was the main hypothesis as to how MAP antigens initiate or accelerate the autoimmune responses, which led to specific CNS damage, suggesting that autoimmunity is triggered by a combination of genetic predisposition and environmental factors such as MAP and Epstein-Barr virus”
\end{abstract}

First draft submitted: 7 April 2019; Accepted for publication: 26 April 2019; Published online:

31 May 2019

Keywords: BCG • etiology • Japan • multiple sclerosis $\bullet$ Mycobacterium avium subsp. paratuberculosis $\bullet$ Sardinia

Multiple sclerosis (MS) is an immune-mediated disease of the central nervous system (CNS) characterized by demyelination and axonal degeneration [1]. It is widely believed that a combination of host genetic factors and exposure to environmental triggers contribute to disease induction or evolution [1]. Several species of mycobacteria, most of which are obligate intracellular pathogens, have been associated with many immune-mediated disorders including MS [1,2]. Multiple studies have suggested a possible causal relationship between Mycobacterium avium subsp. paratuberculosis (MAP) and MS [1-3]. MAP is an obligate intracellular bacterium belonging to Mycobacterium avium complex, it is the causative agent of Johne's disease (paratuberculosis) in ruminants, and it has also been associated with several human autoimmune disorders such as Crohn's disease, Type 1 diabetes and MS [2].

\section{Initial evidence from Sardinia}

The association between MAP and MS was proposed for the first time in 2010 in MS patients from Sardinia, a Mediterranean Italian island characterized by a high incidence of the disease (330 per 100,000 people), and by the endemic presence of MAP (60\% of sheep herds are infected by the bacilli) [4]. Briefly, the association between MAP and MS was initially based on two key findings: the detection of MAP DNA in the peripheral blood of $25 \%$ of MS patients compared with $4 \%$ of healthy subjects, and the discovery of several MAP-specific antigens that share sequence homology with MS-related human proteins which are capable of eliciting a significantly stronger humoral and cell-mediated response in both sera and cerebrospinal fluid (CSF) of MS patients compared with controls [4-6]. For this reason, molecular mimicry was the main hypothesis as to how MAP antigens initiate or accelerate the autoimmune responses, which led to specific CNS damage [4], suggesting that autoimmunity is triggered by a combination of genetic predisposition and environmental factors such as MAP and Epstein-Barr virus [7]. Indeed, competitive assays have shown that peptides from different pathogens can be cross-recognized by antibodies targeting self-epitopes, indicating that Epstein-Barr virus and MAP might cause autoimmunity through a common target [6-8]. In previous work, Cossu et al. reviewed the association with MAP and MS in Sardinia and a hypothetic course of action of the bacteria [3].

\section{Evidence from Japan}

Globally, the incidence and prevalence of MS vary substantially, and the mechanisms underlying these conundrums in geographical incidence of MS are unknown. MS is most common in the western world and in contrast, is uncommon in Asia [9]. However, epidemiological studies have revealed the greatest increase in newly diagnosed MS patients in Japan (crude incidence rate is 15 per 100,000 people) [10]. Genetic changes cannot solely explain

Future $\because$ Medicine 
such rapid rates of increased incidence, so is it possible that the presence of some exclusive environmental factors may modify the prevalence of the disease in Japan. Momotani reported in 2012 that the prevalence of bovine paratuberculosis in Japan was only $2 \%$, in contrast to the highest incidence in western countries [11]. However, keeping in view the increasing prevalence of MAP, no detailed recent studies on prevalence of paratuberculosis have been attempted in Japan.

Otsubo et al. measured the serum levels of total IgG and subclasses targeting MAP surface antigens in 136 Japanese healthy subjects, detecting IgG1 and IgG4 antibodies against MAP antigen in 14\% of the population [12]. The results obtained represented the first piece of evidence implying that the Japanese population was exposed to MAP antigens. Since fecal-oral transmission is considered the first route of transmission from animal to human, the exposure to heath-killed MAP antigenic components was likely due to the consumption of dairy products imported from western countries [12]. 1 year later, in an international collaboration between Juntendo University and Sassari University, Cossu et al. performed an analysis of the humoral response mounted against host-mimetic homologous peptides of MAP and MS in Japanese patients with inflammatory demyelinating diseases of the CNS [13]. A strong, statistically significant humoral response was detected in the sera of relapsing-remitting MS patients only against MAP_2694295-303 peptide. The latter is a surface-exposed epitope of an MAP-specific transmembrane protein and displays sequence homology with the $\mathrm{C}$ region of the TCR $\gamma$ chain expressed by $\gamma \delta \mathrm{T}$ cells $[4,14]$. Kumar et al., using a molecular modeling approach, observed that MAP_2694295-303 peptide displays a preferential binding to the HLA proteins associated with MS $\left({ }^{*} 1501,{ }^{*} 0301\right)$, showing a higher probability of T-cell activation which could be involved in the development of autoimmune diseases [15]. The prevalence of antibodies against MAP_2694295-303 peptide in MS Japanese subjects (30\%) [14] was essentially similar to that in Italian study (35\%) [5], highlighting the importance of MAP_2694 295-303 as an immunogenic peptide that likely plays a role in determining immunogenicity in patients with a genetic susceptibility to the mycobacterium. Of note, HLA DPB ${ }^{*} 0301$, and in particular HLA DRB $1 * 0405$ alleles, are associated with a greater risk of developing MS in a few countries such as Sardinia and Japan [13]. A genetic study conducted by Frau et al. revealed that Sardinian MS patients showed a presence of ${ }^{*} 03: 01-{ }^{*} 02: 01$ and ${ }^{*} 04: 05-{ }^{*} 03: 01$ haplotypes in 36 and 6\% of the MAP_2694-positive subjects, respectively [16]. On the other hand, the discrepancies in antibody determination between the Japanese and Sardinian populations suggested that MS patients from different ethnic backgrounds have an altered immune response to MAP antigens. The genetic background, the differing assessment of human exposure to the mycobacterium and the different coverage rates of Bacillus Calmette-Guérin (BCG) vaccination between populations are all important determinants of susceptibility to the disease. Regarding BCG, clinical trials have shown a vaccine-associated reduction in symptom severity among patients with MS and clinical isolated syndrome [17], however, the mode of action has not yet fully elucidated.

In a recent retrospective study, a total of 97 Japanese patients affected by inflammatory demyelinating disorders of the CNS and all BCG vaccinated, were tested for the detection of total IgG against MAP and BCG surface antigens by indirect ELISA [17]. The study provided more evidence supporting the causative role of MAP, while BCG seemed to be inversely related to the risk of developing MS.

Yokoyama et al. detected higher serum levels of anti-MAP_2694 $295-303$ antibodies in paired CSF and serum samples from Japanese patients with MS when compared with patients with neuromyelitis optica spectrum disorder and controls [18]. Moreover, a group of patients with MS showed intrathecally restricted production of antibodies against MAP_2694295-303 peptide. Women appeared to mount a stronger humoral response to mycobacterial antigens than men, suggesting sex-related differences in the immune response to MAP [18]. In the same work, a strong humoral response against MAP pentapeptide (MAP_5p) was also detected in both sera and CSF of patients with MS. MAP_5p is a cell-wall antigenic component able to discriminate MAP from other nontuberculosis pathogenic mycobacteria [18].

\section{Evidence from animal studies}

Not only human studies, but also new animal experiments, have revealed the adjuvant property of MAP in experimental autoimmune encephalomyelitis (EAE), a murine model of MS characterized by perivascular $\mathrm{CD}_{4}^{+} \mathrm{T}$-cell and mononuclear cell inflammation and subsequent primary demyelination of axonal tracks in the CNS [19]. Groups of C57BL/6 mice were actively immunized with $\mathrm{MOG}_{35-55}$ peptide emulsified in incomplete Freund's adjuvant modified to contain heat-killed MAP. These groups demonstrated an early disease onset and more severe clinical scores in comparison with classical immunization with complete Freund's adjuvant containing Mycobacterium tuberculosis [19]. These findings indicated the existence of species-specific antigens that recognize distinct pattern 
recognition receptors. Several antigenic components present in the complex mycobacterial cell wall are responsible for Toll-like receptor (TLR) activation, which plays an important role in orchestrating proinflammatory and innate immune responses. TLRs are a major driving force for the immune response necessary to develop EAE [19], and it has been shown that MAP components stimulate TLR2, TLR4 and TLR9 on immune cells [20]. At present, the interaction of MAP with the innate immune system, as well the role of the receptors involved in its recognition is not yet fully elucidated.

Ongoing studies are analyzing the efficacy of heath-killed MAP immunization through the oral route, compared with live BCG immunization via intradermal vaccination in the development of EAE, as well the in vivo effect of MAP by using spontaneous EAE murine TCR-transgenic models (specific for $\mathrm{MOG}_{35-55}$ peptide), in order to simulate the clinical and pathological complexity of human MS.

\section{Conclusion}

Although these experimental observations from clinical and animal studies have confirmed the association and provided new data to the puzzle of MAP or BCG and MS, it is still unknown whether a causal link exists. More research is needed to further explore specific aspects of host-pathogen interplay in the physiopathology and treatment of immuno-mediated disorders, combining data obtained from animal models and epidemiological studies.

\section{Financial \& competing interests disclosure}

The authors have no relevant affiliations or financial involvement with any organization or entity with a financial interest in or financial conflict with the subject matter or materials discussed in the manuscript. This includes employment, consultancies, honoraria, stock ownership or options, expert testimony, grants or patents received or pending, or royalties.

No writing assistance was utilized in the production of this manuscript.

\section{References}

Papers of special note have been highlighted as: • of interest; $\bullet \bullet$ of considerable interest

1. Cossu D, Yokoyama K, Hattori N. Bacteria-host interactions in multiple sclerosis. Front. Microbiol 9, 2966 (2018).

2. Cossu D, Yokoyama K, Hattori N. Conflicting role of Mycobacterium species in multiple sclerosis. Front. Neurol. 8, 216 (2017).

3. Cossu D, Masala S, Sechi LA. A Sardinian map for multiple sclerosis. Future Microbiol. 8(2), 223-232 (2013).

4. Cossu D, Cocco E, Paccagnini D et al. Association of Mycobacterium avium subsp. paratuberculosis with multiple sclerosis in Sardinian patients. PLoS ONE 6, e18482 (2011).

- First report of the association between Mycobacterium avium subsp. paratuberculosis (MAP) and multiple sclerosis in the context of Sardinian population.

5. Frau J, Cossu D, Coghe G et al. Mycobacterium avium subsp. paratuberculosis and multiple sclerosis in Sardinian patients: epidemiology and clinical features. Mult. Scler. 19(11), 1437-1442 (2013).

6. Mameli G, Cocco E, Frau J, Marrosu MG, Sech LA. Epstein-Barr virus and Mycobacterium avium subsp. paratuberculosis peptides are recognized in sera and cerebrospinal fluid of MS patients. Sci. Rep. 6, 22401 (2016).

7. Mameli G, Cossu D, Cocco E et al. Epstein-Barr virus and Mycobacterium avium subsp. paratuberculosis peptides are cross recognized by anti-myelin basic protein antibodies in multiple sclerosis patients. J. Neuroimmunol. 270(1-2), 51-55 (2014).

8. Cossu D, Mameli G, Galleri G et al. Human interferon regulatory factor 5 homologous epitopes of Epstein-Barr virus and Mycobacterium avium subsp. paratuberculosis induce a specific humoral and cellular immune response in multiple sclerosis patients. Mult. Scler. 21(8), 984-995 (2015).

9. GBD 2016 Neurology Collaborators. Global, regional, and national burden of neurological disorders, 1990-2016: a systematic analysis for the Global Burden of Disease Study 2016. Lancet Neurol. 18(4), 357-375 (2019).

10. Houzen H, Niino M, Hirotani M et al. Increased prevalence, incidence, and female predominance of multiple sclerosis in northern Japan. J. Neurol. Sci. 323(1-2), 117-122 (2012).

11. Momotani E. Epidemiological situation and control strategies for paratuberculosis in Japan. Jpn J. Vet. Res. 60(Suppl. 3), S19-S29 (2012).

12. Otsubo S, Cossu D, Shigetoshi Eda et al. Seroprevalence of IgG1 and IgG4 class antibodies against Mycobacterium avium subsp. paratuberculosis in Japanese population. Foodborne Pathog. Dis. 12(10), 851-856 (2015).

13. Cossu D, Yokoyama K, Sechi LA et al. Humoral response against host-mimetic homologous epitopes of Mycobacterium avium subsp. paratuberculosis in Japanese multiple sclerosis patients. Sci. Rep. 6, 29227 (2016).

- First report of the association between MAP and multiple sclerosis in the context of Japanese population. 
14. Cossu D, Masala S, Frau J et al. Antigenic epitopes of MAP2694 homologous to T-cell receptor gamma-chain are highly recognized in multiple sclerosis Sardinian patients. Mol. Immunol. 57(2), 138-140 (2014).

15. Kumar A, Sechi LA, Caboni P, Marrosu MG, Atzori L, Pieroni E. Dynamical insights into the differential characteristics of Mycobacterium avium subsp. paratuberculosis peptide binding to HLA-DRB1 proteins associated with multiple sclerosis. N.J. Chem. 39(2), 1355-1366 (2015).

16. Frau J, Cossu D, Sardu C et al. Combining HLA-DRB1-DQB1 and Mycobacterium avium subspecies paratubercolosis (MAP) antibodies in Sardinian multiple sclerosis patients: associated or independent risk factors? BMC Neurol. 16(1), 148 (2016).

17. Cossu D, Yokoyama K, Tomizawa Y, Momotani E, Hattori N. Altered humoral immunity to mycobacterial antigens in Japanese patients affected by inflammatory demyelinating diseases of the central nervous system. Sci. Rep. 7, 3179 (2017).

18. Yokoyama K, Cossu D, Hoshino Y, Tomizawa Y, Momotani E, Hattori N. Anti-mycobacterial antibodies in paired cerebrospinal fluid and serum samples from Japanese patients with multiple sclerosis or neuromyelitis optica spectrum disorder. J. Clin. Med. 7(12), E522 (2018).

19. Cossu D, Yokoyama K, Sakanishi T, Eiichi M, Hattori N. Adjuvant and antigenic properties of Mycobacterium avium subsp. paratuberculosis on experimental autoimmune encephalomyelitis. J. Neuroimmunol. 330, 174-177 (2019).

- First report about the impact of MAP and experimental autoimmune encephalomyelitis.

20. Ferwerda G, Kullberg BJ, de Jong DJ et al. Mycobacterium paratuberculosis is recognized by Toll-like receptors and NOD2. J. Leukoc. Biol. 82(4), 1011-1018 (2007). 